\title{
SWOT of Bigdata Security Using Machine Learning Techniques
}

\author{
Ravikumar Muljibhai Rohit \\ SMT. Chandaben Mohanbhai Patel Institute of Computer Applications, Changa. \\ Charotar University Of Science And Technology, Changa, India
}

\begin{abstract}
This paper gives complete guidelines on BigData, Different Views of BigData, etc.How the BigData is useful to us and what are the factors affecting BigData all the things are covered under this paper. The paper also contains the BigData Machine learning techniques and how the Hadoop comes into the picture. It also contains the what is importance of BigData security. The paper mostly covers all the main point that affect Big Data and Machine Learning.
\end{abstract}

\section{KEYWORDS}

BigData, BigData Security, Machine Learning, Threats of BigData, Hadoop etc.

\section{INTRODUCTION}

"Big Data" well-known word sounds around us. But only few are aware about it, we only knows that Big Data means Large amount of data but it is not just amounts of data only, it is more than that.

The term big data has come into use recently to refer to the ever-increasing amount of information that organizations are storing, processing and analyzing, owing to the growing number of information sources in use. According to research conducted by IDC ([10].IDC Aalyze the Future n.d.)there were 1.8 zettabytes (1.8 trillion gigabytes) of information created and replicated in 2011 alone and that amount is doubling every two years. 1 Within the next decade, the amount of information managed by enterprise datacenters will grow by $\mathbf{5 0}$ times, whereas the number of IT professionals will expand by just 1.5 times. The process of research into massive amounts of data to reveal hidden patterns and secret correlations named as big data analytics. These useful information's for company's helps of gaining richer and deeper insights and getting an advantage over the competition. For this reason, big data implementations need to be analyzed and executed as accurately as possible.

\section{TIME LINE}

\begin{tabular}{|l|l|l|}
\hline $\begin{array}{l}\text { Sr. } \\
\text { No. }\end{array}$ & Era/Year & Short Description \\
\hline 1 & $\begin{array}{l}\text { C } 18,000 \\
\text { BCE }\end{array}$ & $\begin{array}{l}\text { Tally sticks are used to record data for the first time to track trading } \\
\text { activity and record inventory. }\end{array}$ \\
\hline 2 & $\begin{array}{l}\text { C } 2400 \\
\text { BCE }\end{array}$ & The abacus is developed and the first libraries are built in Babylonia. \\
\hline
\end{tabular}




\begin{tabular}{|c|c|c|}
\hline 3 & \begin{tabular}{|l|}
$300 \mathrm{BCE}-$ \\
$48 \mathrm{AD}$
\end{tabular} & $\begin{array}{l}\text { The library of Alexandria is the world's largest data storage center -unit } \\
\text { later on destroyed by the Romans. }\end{array}$ \\
\hline 4 & $\begin{array}{l}100 \mathrm{AD}- \\
200 \mathrm{AD}\end{array}$ & $\begin{array}{l}\text { The AntikytheraMechanism([2].Antikythera mechanism n.d.):- the first } \\
\text { mechanical computer is developed in Greece. }\end{array}$ \\
\hline 5 & 1663 & $\begin{array}{l}\text { John Graunt conducts the first recorded statistical-analysis experiments in } \\
\text { an attempt to curb the spread of the bubonic plague in Europe. }\end{array}$ \\
\hline 6 & 1865 & $\begin{array}{l}\text { The term "Business Intelligence" is used by Richard miller Devens in his } \\
\text { encyclopedia of Commercial and Business Anecdotes }\end{array}$ \\
\hline 7 & 1881 & $\begin{array}{l}\text { Herman Hollerith creates the Hollerith Tabulating Machine which uses } \\
\text { punch cards to reduce the workload of the US Census. }\end{array}$ \\
\hline 8 & 1926 & $\begin{array}{l}\text { Nikola Tesla Predicted that in the feature, man will be able to access and } \\
\text { analyze vast amount of data using a device small enough to fit in his } \\
\text { pocket. }\end{array}$ \\
\hline 9 & 1928 & $\begin{array}{l}\text { Fritz Pfleumer creates a method of storing data magnetically, which forms } \\
\text { basic of modern digital data storage technology }\end{array}$ \\
\hline 10 & 1944 & $\begin{array}{l}\text { Fremont Rider Speculates that Yale Library will contain } 200 \text { million books } \\
\text { stored on } 6,000 \text { miles of shelves, by } 2040\end{array}$ \\
\hline 11 & 1958 & $\begin{array}{l}\text { Hans peter Luhn defines BI as "the ability to apprehend the } \\
\text { interrelationships of presented facts in such a way as to guide action } \\
\text { towards a desired goal." }\end{array}$ \\
\hline 12 & 1965 & $\begin{array}{l}\text { The US Government plans the world's first data center to store } 742 \text { million } \\
\text { tax returns and } 175 \text { million sets of fingerprints on magnetic tape. }\end{array}$ \\
\hline 13 & 1970 & $\begin{array}{l}\text { Relational Database model developed by IBM ([9]IBM } \\
\text { n.d.)mathematician Edgar F Codd. The Hierarchal file system allows to } \\
\text { access records using a simple index system. }\end{array}$ \\
\hline 14 & 1976 & $\begin{array}{l}\text { Material Requirements Planning (MRP) systems are commonly used in } \\
\text { business. Computer and data storage is used for everyday routine tasks. }\end{array}$ \\
\hline 15 & 1989 & $\begin{array}{l}\text { Early use of term Big Data in magazine article by fiction author Erik } \\
\text { Larson- commenting on advertisers' use of data to target customers. }\end{array}$ \\
\hline 16 & 1991 & $\begin{array}{l}\text { The birth of the internet. Anyone can upload their own data, or analyze } \\
\text { data uploaded by other people. }\end{array}$ \\
\hline 17 & 1996 & $\begin{array}{l}\text { The price of digital storage falls to the point where it is more cost-effective } \\
\text { than paper. }\end{array}$ \\
\hline 18 & 1997 & $\begin{array}{l}\text { Google launch their Search engine which will quickly become the most } \\
\text { popular in the world. }\end{array}$ \\
\hline 19 & 1999 & $\begin{array}{l}\text { First use of the Big Data in academic paper - Visually Exploring Gigabyte } \\
\text { Datasets in Realtime(ACM) ([1].ACM n.d.) }\end{array}$ \\
\hline 20 & 2001 & Three "Vs" of Big Data-Volume Velocity, Variety defined by Doug Laney \\
\hline 21 & 2005 & $\begin{array}{l}\text { Hadoop-an open source Big Data framework now developed by Apache- is } \\
\text { developed. "web } 2.0 \text { came into the market" }\end{array}$ \\
\hline 22 & 2008 & $\begin{array}{l}\text { Globally } 9.57 \text { trillion gigabytes of information is processed by the world's } \\
\text { CPUs. }\end{array}$ \\
\hline 23 & 2009 & S company with over 1,000 employees is storing more than \\
\hline
\end{tabular}


International Journal of Information Sciences and Techniques (IJIST) Vol.6, No.1/2, March 2016

\begin{tabular}{|l|l|l|}
\hline & & 200 terabytes of data according to the report Big Data. \\
\hline 24 & 2010 & $\begin{array}{l}\text { Eric Schmidt, executive chairman of Google, tells at conference that as } \\
\text { huge data is now being created from the beginning of human civilization to } \\
\text { the year 2003. }\end{array}$ \\
\hline 25 & 2011 & $\begin{array}{l}\text { The McKinsey report states that by 2018 the US will face a loss of between } \\
140,000 \text { and } 190,000 \text { professional data scientists, and warns on issues } \\
\text { including privacy, security and intellectual property will have to be } \\
\text { resolved. }\end{array}$ \\
\hline 26 & 2014 & $\begin{array}{l}\text { Mobile internet overtakes desktop for the first time, } 88 \% \text { of executives } \\
\text { responding to an international survey by GE state that big data analysis is a } \\
\text { top priority }\end{array}$ \\
\hline
\end{tabular}

\section{THREE DIFFERENT VIEWS OF BIG DATA}

Data mainly expanding on Three Views (fronts/views) at an increasing rate.

\subsection{Data Volume}

\subsection{Data Velocity}

\subsection{Data Variety}

\subsection{Data Volume}

General meaning of volume is the amount of space that a substance or object occupies. As we relate with data volume the unstructured data streaming in from social media increasing rapidly required a large volume space. Increasing amounts of sensor and machine-to-machine data being collected. In the past, excessive data volume was a storage issue. But with decreasing storage costs, other issues emerge, including how to determine relevance within large data volumes and how to use analytics to create value from relevant data.

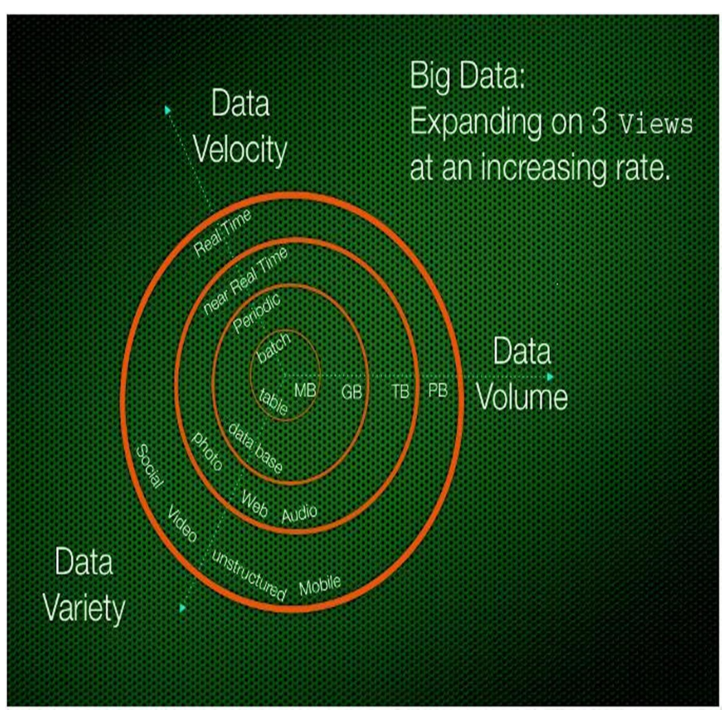


International Journal of Information Sciences and Techniques (IJIST) Vol.6, No.1/2, March 2016

\subsection{Data Velocity}

In general term velocity is the speed of something in a given direction. Data is streaming in at unprecedented speed and must be dealt with in a timely manner. RFID tags (Radio-frequency identification), sensors and smart metering are driving the need to deal with torrents of data in near-real time. Dealing with data velocity is a challenge for most organizations.

\subsection{Data Variety}

Data today comes in all types of formats. Structured, numeric data in traditional databases. Unstructured text documents, email, video, audio, stock ticker data and financial transactions. Managing, merging and governing different varieties of data are vast scope to gather information from, many organizations still struggle with.

Now days there are two more additional dimensions has come when thinking about big data

\subsection{Data Variability}

Variability means inconsistency in addition to the increasing velocities and varieties of data, data flows can be highly inconsistent with periodic peaks.

\subsection{Data Complexity}

Today's data comes from multiple sources. It is still an undertaking to link, match, cleanse and transform data across systems. However, it is necessary to connect and correlate relationships, hierarchies and multiple data linkages or your data can quickly spiral out of control.

\section{Big Data Security}

Data volumes continue to expand as they take in an ever-wider range of sources, much of which is in unstructured form. Many organizations want to extract value from that data to uncover the opportunities for the business that it contains. But the centralized nature of big data stores creates new security challenges.

Traditional tools are not up to the task of processing the information the data contains, let alone ensuring it's secure in the process. Colin Tankard of Digital Pathways (Enterprice level security n.d.) explains that controls need to be placed around the data itself, rather than the applications and systems that store the data.

\section{Security methods for big data}

\subsection{Type Based Keyword Search for Security Of Big Data. 5.1.1System Model}

We will design a secure big data storage system that supports multiple users. In this system, authorized users are able to store encrypted data and carry out keyword queries on encrypted data without decrypting all the files. Moreover, data owners could delegate certain type of files to other users. 
International Journal of Information Sciences and Techniques (IJIST) Vol.6, No.1/2, March 2016

\subsubsection{Security Analysis}

We discuss our type based keyword search for encrypted data from the following security requirements: data confidentiality and query privacy. We assume that users "e private keys are kept secret.

\section{Top most challenges:}

6.1. Secure computations in distributed programming frameworks

6.2. Security best practices for non-relational data stores

6.3. Secure data storage and transactions logs

6.4. End-point input validation/filtering

6.5. Real-Time Security Monitoring

6.6. Scalable and compostable privacy-preserving data mining and analytics

6.7. Cryptographically enforced data centric security

6.8. Granular access control

6.9. Granular audits

\section{Data set for big data}

A data set is a named collection of data that contains individual data units formatted in a specific, there are many way and accessed by a specific access method that is based on the data set organization. Types of data set organization include

\subsection{Sequential}

\subsection{Relative sequential}

\subsection{Indexed sequential}

\subsection{Partitioned}

Access methods include:

\subsection{Virtual Sequential Access Method ( VSAM )}

\subsection{Indexed Sequential Access Method ( ISAM ).}

\section{Dataset freely available}

Cross-disciplinary data repositories, data collections and data search engines which are freely available most popular dataset are as follow. 
International Journal of Information Sciences and Techniques (IJIST) Vol.6, No.1/2, March 2016

\subsection{USGOV XML Dataset}

US Government Dataset which is freely available ([17].us Government n.d.)

\subsection{Amaxon}

Amazon Dataset ([18].Amazon dataset n.d.)

\section{Big Data Attributes}

There are mainly 5 Attributes of a Big Data Strategy

\subsection{Business Case}

To improve real-time reporting and predictive analysis.

\subsection{Architecture}

It covers Ingestion/extraction job control, data storage area, refinery \& data prediction, security, metadata, analytical, data discovery, BI, model execution tool, HW Platform, Hadoop distribution/targeted release.

\subsection{Skill Development}

We must have our own patterns, technique and algorithms to handle the big data.

\subsection{Governance}

Data governance must be exception base.

\subsection{Big Data POC}

Work through architect details, provide a plan based on real-world experience, Test BI/Data Discovery, and provide Sizing information, business use-case validation.

\section{Threats of big data}

Security Intelligence with Big Data solution will empower an organization to address the needs of a changing security landscape. The following are categories of use cases where it can prove at least beneficial if not essential

\subsection{Establish a Baseline}

Organization gains an understanding of its ecosystem, what needs to be defended or observed as well as formulating a risk profile enabling it to detect abnormalities.

Common Use Case Questions:

Who are the attractive targets within any enterprise?

Which applications and what data do someone need to defend due to their sensitivity?

What is the normal behavior profile for users, assets, and applications? 
International Journal of Information Sciences and Techniques (IJIST) Vol.6, No.1/2, March 2016

\subsection{Recognize Advanced Persistent Threats:}

Organization gains awareness of a motivated or incentivized attacker who attempts to hide or disguise the attack as innocuous interactions, potentially over a long period of time (months, years).

Common Use Case Questions:

Which external domains may be the source of attacks?

Are there any low profile network traffic elements that might signal an ongoing or imminent attack?

\subsection{Qualify Insider Threats}

Organization gains evidence or is warned of users within the organization's network who may be inclined to steal intellectual property, compromise enterprise systems or perform other actions that are detrimental to the organization's operations.

Common Use Case Questions:

What data is being leaked or lost and by whom?

Who internally has the motivation and skills to compromise the cyber operations of the company?

Who is exhibiting abnormal usage behavior?

\subsection{Predict Hacktivism}

Organization is alerted to attack from groups or entities that sympathize with causes that are contrary to the business interests of an enterprise.

Common Use Case Questions:

Which controversial issues may trigger a negative sentiment about the organization triggering an increased risk of attack?

How to identify and monitor intentions of entities antagonistic to the organization's business practices?

How does publicity of the company in the media impact risk?

\subsection{Counter Cyber Attacks}

Organization is informed of an impending or on-going attack by criminal enterprises or government funded or government sponsored groups.

Common Use Case Questions:

What is the origin of an attack?

Which hacking tools may be used and who is gaining access to them? 
International Journal of Information Sciences and Techniques (IJIST) Vol.6, No.1/2, March 2016

Are their symptoms of an attack underway or being planned manifesting themselves as support issues?

\subsection{Mitigate Fraud}

Organization is appraised of new or existing fraud methods that may compromise its compliance with regulations or cause significant losses to its financial operations.

Common Use Case Questions:

How can the organization identify a fraudulent activity?

Which users have compromised identities that may lead to fraudulent activity?

Can well known fraud attempts have patterns can either be detected or even anticipated?

\section{Solution}

Combating Advanced Threats with Security Intelligence

A good security intelligence solution enables complex problem-solving capabilities, uniquely equipping them to defend against advanced threats. Let's look at critical capabilities of effective security intelligence solutions.

\subsection{Consolidation of data silos for 360-degree view}

Connect the dots between seemingly unrelated or benign activities and ultimately deliver better insight for advanced threat detection.

\subsection{Pre- and post-exploit insights}

Gather and prioritize information about existing security gaps to prevent breaches, as well as suspicious behavior to detect breaches.

\subsection{Forensic capabilities}

Exhaustively research the impact of the breach using captured packet data, easing the burden on the security and network staff who have to build a remediation plan.

\subsection{Anomaly detection capabilities}

Baseline current activity and identify meaningful deviations - a core and vital aspect of detecting advanced threats in progress.

\subsection{Real-time correlation and analysis}

Process massive data sets using advanced analytical methods and purpose-built data repositories, allowing for earlier and more accurate detection of advanced threats, and helping to distinguish the signal from the noise. 


\subsection{Helping reduce false positives}

De-prioritize unusual yet benign activity to reduce the time spent investigating anomalous but harmless activity, helping the organization focus on its top incidents.

\subsection{Flexibility}

Constant environmental changes require constant product evolution to add data sources, create and tune analytics, create new user views and reports, and expand and evolve the overall deployment architecture.

\subsection{Unified approach}

Prevention of complex, multi-pronged attacks requires a unified or integrated platform to help organizations intelligently wade through hundreds of security alerts and massive quantities of raw event and flow data.

\section{Machine Learning}

"Big Data" and "Machine Learning" as connected activities. People have been talking about the need for more 'analysis' and insight in big data, which is obviously important, because we've been in the 'collection' phase with big data until now. But the innovation in the big data world that I'm most excited about is the 'prediction' phase - the ability to process the information we've collected, learn patterns, and predict unknowns based on what we've already seen.

Machine learning is to big data as human learning is to life experience: We interpolate and extrapolate from past experiences to deal with unfamiliar situations. Machine learning with big data will duplicate this behaviour, at massive scales.

\section{Big Data needs Big Compute: Where Hadoop and Spark fit in the picture}

Think of big data and machine learning as three steps:

\subsection{Collect}

\subsection{Analyze}

\subsection{Predict}

These steps have been disconnected until now, because we've been building the ecosystem from the bottom up - experimenting with various architectural and tool choices - and building a set of practices around that.

The early Hadoop stack is an example of collecting and storing big data. It allows easier data processing across a large cluster of cheap commodity servers. But Hadoop MapReduce is a batchoriented system, and doesn't lend itself well towards interactive applications; real-time operations like stream processing; and other, more sophisticated computations. 
International Journal of Information Sciences and Techniques (IJIST) Vol.6, No.1/2, March 2016

For predictive analytics, we need an infrastructure that's much more responsive to human-scale interactivity: What's happening today that may influence what happens tomorrow? A lot of iteration needs to occur on a continual basis for the system to get smart, for the machine to

"learn" - explore the data, visualize it, build a model, ask a question, an answer comes back, bring in other data, and repeat the process.

The more real-time and granular we can get, the more responsive, and more competitive, we can be.

Compare this to the old world of "small-data" business intelligence, where it was sufficient to have a small application engine that sat on top of a database. Now, we're processing a thousand times more data, so to keep up the speed at that scale, we need a data engine that's in-memory and parallel. And for big data to unlock the value of machine learning, we're deploying it at the application layer. This means "big data" needs "big compute".

This is where Apache Spark comes in. Because it's an in-memory, big-compute part of the stack, it's a hundred times faster than Hadoop MapReduce. It also offers interactivity since it's not limited to the batch model. Spark runs everywhere (including Hadoop), and turns the big data processing environment into a real-time data capture and analytics environment.

\section{Five major advantages of Hadoop 14.1. Scalable}

Hadoop is a highly scalable storage platform, because it can store and distribute very large data sets across hundreds of inexpensive servers that operate in parallel. Unlike traditional relational database systems (RDBMS) that can't scale to process large amounts of data, Hadoop enables businesses to run applications on thousands of nodes involving thousands of terabytes of data.

\subsection{Cost effective}

Hadoop also offers a cost effective storage solution for businesses' exploding data sets.

The problem with traditional relational database management systems is that it is extremely cost prohibitive to scale to such a degree in order to process such massive volumes of data. In an effort to reduce costs, many companies in the past would have had to down-sample data and classify it based on certain assumptions as to which data was the most valuable. The raw data would be deleted, as it would be too cost-prohibitive to keep. While this approach may have worked in the short term, this meant that when business priorities changed, the complete raw data set was not available, as it was too expensive to store. Hadoop, on the other hand, is designed as a scale-out architecture that can affordably store all of a company's data for later use (htt). The cost savings are staggering: instead of costing thousands to tens of thousands of pounds per terabyte, Hadoop offers computing and storage capabilities for hundreds of pounds per terabyte.

\subsection{Flexible}

Hadoop enables businesses to easily access new data sources and tap into different types of data (both structured and unstructured) to generate value from that data. This means businesses can use Hadoop to derive valuable business insights from data sources such as social media, email conversations or click stream data. In addition, Hadoop can be used for a wide variety of purposes, such as log processing, recommendation systems, data warehousing, market campaign analysis and fraud detection. 
International Journal of Information Sciences and Techniques (IJIST) Vol.6, No.1/2, March 2016

\subsection{Fast}

Hadoop's unique storage method is based on a distributed file system that basically 'maps' data wherever it is located on a cluster. The tools for data processing are often on the same servers where the data is located, resulting in much faster data processing.

If you're dealing with large volumes of unstructured data, Hadoop is able to efficiently process terabytes of data in just minutes, and petabytes in hours.

\subsection{Resilient to failure}

A key advantage of using Hadoop is its fault tolerance. When data is sent to an individual node, that data is also replicated to other nodes in the cluster, which means that in the event of failure, there is another copy available for use (Hadoop n.d.).

The MapR distribution goes beyond that by eliminating the NameNode and replacing it with a distributed No NameNode architecture that provides true high availability. Our architecture provides protection from both single and multiple failures.

When it comes to handling large data sets in a safe and cost-effective manner, Hadoop has the advantage over relational database management systems, and its value for any size business will continue to increase as unstructured data continues to grow.

Michele Nemschoff is vice president of corporate marketing at MapR Technologies.

\section{Conclusion}

The availability of Big Data, low-cost commodity hardware, and new information management and analytic software has produced a unique moment in the history of data analysis. The convergence of these trends means that we have the capabilities required to analyze astonishing data sets quickly and cost-effectively for the first time in history. These capabilities are neither theoretical nor trivial. They represent a genuine leap forward and a clear opportunity to realize enormous gains in terms of efficiency, productivity, revenue, and profitability. The Age of Big Data is here, and these are truly revolutionary times if both business and technology professionals continue to work together they can achieve a great benefit.

\section{ACKNOWLEDGEMENTS}

I would like to thank our College (CMPICA), department and as specially Mr. Kanubhai Patel (Pro. At CMPICA, CHARUSAT Changa.) Who have been so helpful to me and guiding me, giving feedback on the paper, without his help it is so hard to complete the paper in time. I am thankful to him and our college.

\section{REFERENCES}

[1] Lee, S.hyun. \& Kim Mi Na, (2008) “This is my paper”, ABC Transactions on ECE, Vol. 10, No. 5, pp120-122.

[2] Gizem, Aksahya \& Ayese, Ozcan (2009) Coomunications \& Networks, Network Books, ABC Publishers.

[3]. ACMhttp://india.acm.org/ 
International Journal of Information Sciences and Techniques (IJIST) Vol.6, No.1/2, March 2016

[4]. Antikythera mechanismhttps://en.wikipedia.org/wiki/Antikythera mechanism

[5]. apacheHadoophttp://www.mapr.com/products/apache-hadoop

[6]. Big data1http://www.sas.com/en_us/insights/big-data/what-is-big-data.html

[7]. Enterprice level securityhttp://digpath.co.uk/

[8]. Hadoophttp://www.mapr.com/products/apache-hadoop

[9]. Hadoophttps://www.mapr.com/products/apache-hadoop

[10]. History of big data: http://www.slideshare.net/BernardMarr/a-brief-history-of-big-data

[11]. IBMwww.ibm.com/in-en/

[12]. IDC Aalyze the Futurehttps://www.idc.com/

[13]. illuminatedhttp://hadoopilluminated.com/hadoop_illuminated/ Public_Bigdata_Sets.html

[14]. machine learningwww.a16z.com/2015/01/22/machine-learning-big-data/

[15]. Security on Bigdatahttp://www.computerweekly.com/feature/How-to-tackle-big-data-from-asecurity-point-of-view

[16]. threatshttps://securityintelligence.com/security-intelligence-with-big-data-what-you-need-toknow/

[17]. ThreeViewshttp://searchcloudcomputing.techtarget.com/definition/big-data-Big-Data

[18]. Usecasehttp://www.vormetric.com/data-security-solutions/use-cases/big-data-security

[19]. Amazon dataset. http://aws.amazon.com/datasets.

\section{Authors}

Ravikumar Muljibhai Rohit

Short Biography

I am pursuing my MCAL $\left[4^{\text {th }} \mathrm{SEM}\right]$ from Smt.

Chandaben Mohanbhai Patel Institute of

Computer Applications

CHARUSAT, Changa.

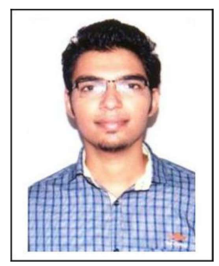

